\title{
La interacción de materiales del sistema sílice-alúmina con productos fluorados aluminosos
}

\author{
L.F. Verdeja ${ }^{(*)}$, S. García ${ }^{(*)}$, A.A. Fernández ${ }^{(*)}$ y R. Corzo ${ }^{(* *)}$
}

\begin{abstract}
Resumen: Este trabajo pretende contribuir al estudio sistemático de los mecanismos de corrosión que experimentan los refractarios sílico-aluminosos, aluminosos y de alta alúmina con productos fluorados y aluminio en estado líquido. Se aportan datos termodinámicos que confirman el papel preponderante del ataque químico y de los mecanismos erosivos en el desgaste de materiales del sistema sílice-alúmina. Se discute, asimismo, la influencia de la composición de las fases cristalinas encontradas en el grado de agresión a los revestimientos cerámicos. Se propone, ante la agresividad de agentes químicamente activos, como la criolita o el fluoruro de aluminio, y la gran afinidad del aluminio por el oxígeno, no aconsejar la utilización de materiales oxídicos y nitrurados de alto valor para el revestimiento de hornos y bolsas de transporte de aluminio. Se propone, como línea de trabajo a seguir, la utilización de materiales de base corindón como los más adecuados para interaccionar con productos fluorados y aluminio en estado líquido, así como estudiar la adherencia de costras como mecanismo de protección del revestimiento del refractario.
\end{abstract}

Palabras clave: Corrosión y erosión de refractarios. Revestimientos cerámicos. Corindon.

\section{Interaction between silica-alumina system materials with fluoride rich alumina products}

\begin{abstract}
The aim of this paper is to get a first approximation of the systematic study of the corrosion mechanisms of the silica-alumina, alumina and rich-alumina refractories by fluoride compounds and molten aluminum. The thermodynamic results support the main role of the chemical attack and the erosive mechanisms in the wear of the silica-alumina materials. The influence of the composition of the crystalline phases in the degree of the ceramic lining attack is also studied. The results suggest not to use either oxide or nitride high-value materials for the lining of furnaces and transportrecipients containing molten aluminum, because of the chemical corrosion by compounds such as cryolite or aluminum fluoride and also, because of the avidity of aluminum towards oxygen. The study of corundum-based materials like the best refractories used in order to react with fluoride compounds and molten aluminum is an alternative to be considered. The study of the crusts adhesion like a protection mechanism of the refractory lining is also a posible approach.
\end{abstract}

Keywords: Refractories corrosion and erosion. Ceramics linings. Corundum.

\section{INTRODUCCIÓN}

El conocimiento de los mecanismos que intervienen fundamentalmente en la degradación de material refractario no ha de considerarse como un mero ejercicio teórico-especulativo de algunos profesionales de la ciencia y de la investigación. Al

(*) E.T.S.I. de Minas. Cátedra de Siderurgia. Univ. de Oviedo. Oviedo (España).

(**) Fac. de Química. Dpto. de Química Física y Analítica. Univ. de Oviedo. Oviedo (España). contrario, el conocimiento de las causas que controlan el desgaste del material puede constituir una herramienta de gran utilidad tanto para los fabricantes como para los usuarios en la optimización de las variables calidad-precio-duración (1).

No obstante, es frecuente identificar la variable calidad con el precio, tal y como sucede con numerosos productos del mercado. Es decir, en el caso de materiales refractarios es corriente afirmar que la solución a un problema de desgaste es, instalar como alternativa, un material de superior calidad con el correspondiente aumento de precio. 
Particularmente, dentro de los materiales del sistema $\mathrm{SiO}_{2}-\mathrm{Al}_{2} \mathrm{O}_{3}$, con altas cotas de utilización en el mercado, es usual resolver los problemas de corrosión y desgaste pensando siempre en una alternativa con mayor contenido de alúmina.

Con independencia de que, desde el punto de vista teórico, en el proceso de corrosión de un refractario puedan intervenir mecanismos abrasivos, erosivos, químicos, adhesivos y mecánico-térmicos, este trabajo se ceñirá al estudio de las interacciones químicas que puedan existir entre los componentes elementales y compuestos a que da lugar el sistema $\mathrm{SiO}_{2}-\mathrm{Al}_{2} \mathrm{O}_{3}$ (Fig. 1) con el propio aluminio metal junto con productos fluorados de distinta naturaleza (2).

\section{TERMODINÁMICA DE LOS PROCESOS DE CORROSIÓN}

El aluminio es un metal que, para desplazarlo de sus combinaciones con otros elementos del sistema periódico, se necesita o bien un metal más activo que el propio aluminio o el concurso de la energía eléctrica. Por otra parte, las combinaciones de productos fluorados de aluminio y alcalinos presentan afinidades eutécticas que dan lugar a la formación de fases líquidas, incluso a temperaturas inferiores a $700{ }^{\circ} \mathrm{C}$.

Es decir, que para temperaturas de trabajo del orden de $900^{\circ} \mathrm{C}$, tanto el aluminio como los eutécticos fluorados van a ser fases líquidas con una gran capacidad de penetración y corrosión dentro del material refractario.

En la tabla I se presenta la evolución con la temperatura de la energía libre estándar, $\Delta G^{\circ}(T)$, de las posibles reacciones entre los constituyentes-com-

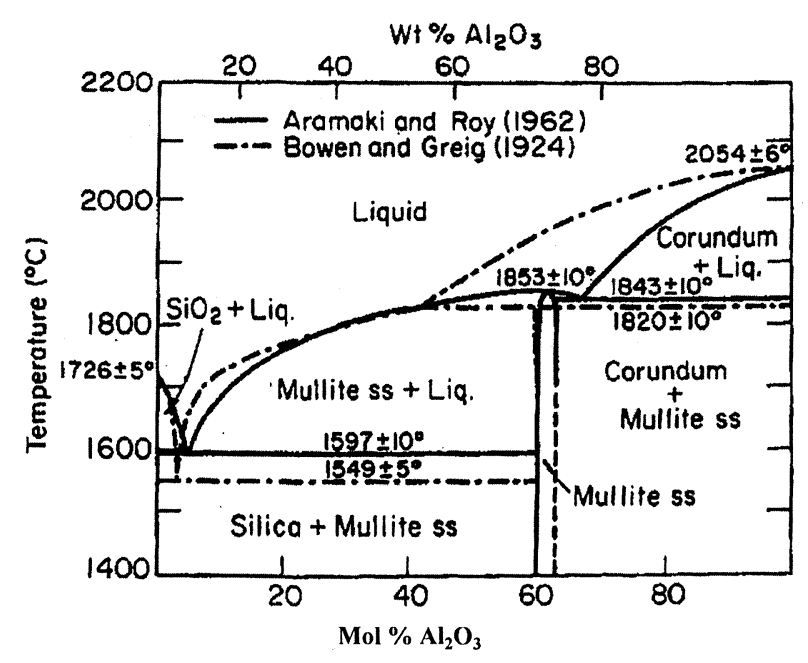

Fig. 1.- Diagrama de fases $\mathrm{SiO}_{2}-\mathrm{Al}_{2} \mathrm{O}_{3}$.

FIG. 1.- Phase diagram $\mathrm{SiO}_{2}-\mathrm{Al}_{2} \mathrm{O}_{3}$.
TABLA I.- Valores de los parámetros de la energía libre y datos de $\Delta \mathrm{G}^{\mathrm{o}}(\mathrm{T})=\mathrm{A}+\mathrm{BT}(\mathrm{J})$

TABLE I.- Parameters of the standard free energy and $\Delta G^{\circ}$ data at $1273 \mathrm{~K}$ for reactions 1-6

\begin{tabular}{|c|r|r|c|}
\hline$\Delta \mathrm{G}^{\mathrm{o}}(\mathrm{T})$ reacción & \multicolumn{1}{|c|}{$\mathrm{A}$} & \multicolumn{1}{c|}{$\mathrm{B}$} & \multicolumn{1}{c|}{$\mathrm{G}^{\mathrm{o}}{ }_{1273 \mathrm{~K}}(\mathrm{~kJ})$} \\
\hline$\Delta \mathrm{G}^{\mathrm{o}}(\mathrm{T})(1)$ & $-6,81 \times 10^{5}$ & 147,0 & $-493,9$ \\
$\Delta \mathrm{G}^{\mathrm{o}}(\mathrm{T})(2)$ & $7,37 \times 10^{5}$ & $-155,0$ & 539,7 \\
$\Delta \mathrm{G}^{\mathrm{o}}(\mathrm{T})(3)$ & $2,82 \times 10^{4}$ & 243,0 & 337,5 \\
$\Delta \mathrm{G}^{\mathrm{o}}(\mathrm{T})(4)$ & $4,63 \times 10^{6}$ & $-378,0$ & $4.148,8$ \\
$\Delta \mathrm{G}^{\mathrm{o}}(\mathrm{T})(5)$ & $-3.00 \times 10^{4}$ & 73,1 & 63,1 \\
$\Delta \mathrm{G}^{\mathrm{o}}(\mathrm{T})(6)$ & $-9,87 \times 10^{4}$ & 107,0 & 37,5 \\
\hline
\end{tabular}

$$
\begin{aligned}
\text { Re acción (1) : } & 3 \mathrm{SiO}_{2(\text { crist.) }}+4 \mathrm{Al}_{1} \leftrightarrow 2 \mathrm{Al}_{2} \mathrm{Q}_{3(\mathrm{~s})}+3 \mathrm{Si}_{\mathrm{s}} \\
\text { Re acción (2) }: & \mathrm{SiO}_{2 \text { (crist.) }}+4 \mathrm{NaF}_{(\mathrm{s} / \mathrm{l})} \leftrightarrow \mathrm{SiF}_{4(\mathrm{~g})}+2 \mathrm{Na}_{2} \mathrm{O}_{(1)} \\
\text { Re acción (3) : } & 2 \mathrm{Al}_{2} \mathrm{O}_{3} \cdot 3 \mathrm{SiO}_{2(\mathrm{~s})}+4 \mathrm{Al}_{(1)} \leftrightarrow \mathrm{Al}_{2} \mathrm{O}_{3(\mathrm{~s})}+3 \mathrm{Si}_{(\mathrm{s})} \\
\operatorname{Re} \text { acción (4) }: & 2 \mathrm{Al}_{2} \mathrm{O}_{3} \cdot 3 \mathrm{SiO}_{2(\mathrm{~s})}+24 \mathrm{NaF}_{(\mathrm{s} / \mathrm{l})} \leftrightarrow 4 \mathrm{AlF}_{3(\mathrm{~s})}+ \\
+ & 3 \mathrm{SiF}_{4(\mathrm{~g})}+12 \mathrm{Na}_{2} \mathrm{O}_{(1)}
\end{aligned}
$$

puestos del sistema sílice-alúmina con el aluminio y el fluoruro de sodio (3):

$$
\Delta G^{\mathrm{o}}(T)=A+B T
$$

Aunque la evolución espontánea e irreversible de un proceso viene determinada por $\Delta G(T), \Delta G^{\circ}$ $(T)$ al indicar el punto en el cual se alcanza el equilibrio y, por tanto, el progreso de la reacción se detiene, marca la pauta del alcance que pueden tener los procesos de ataque químico.

Ciertamente, el ataque de la cristobalita va a ser mucho más violento que el de la mullita al entrar en contacto con el aluminio. Igualmente, puede esperarse un mayor desgaste de la interacción del fluoruro de sodio con la cristobalita que con la mullita.

A la vista de estos primeros resultados, cabía esperar una solución para el contacto de materiales del sistema sílice-alúmina consistente en productos sinterizados a alta temperatura con el objeto de afrontar la sinterización de cristales de mullita sin dar opción a fases líquidas con altos contenidos de sílice. Aunque técnicamente factible, económicamente la solución no es válida al ser la mullita una materia prima sintética de alto valor.

La propia alúmina, $\alpha-\mathrm{Al}_{2} \mathrm{O}_{3}$, presenta una cierta afinidad química por los medios fluorados (4) alcanzando una proporción del $10 \%$ en criolita a $962{ }^{\circ} \mathrm{C}$ (Fig. 2).

Fuera del sistema $\mathrm{SiO}_{2}-\mathrm{Al}_{2} \mathrm{O}_{3}$ se especula que con la formulación de materiales a base de carburo de silicio u óxido de zirconio pudieran ser más resistentes al ataque químico que los componentes derivados del sistema $\mathrm{SiO}_{2}-\mathrm{Al}_{2} \mathrm{O}_{3}$ : la cristobalita, el corindón y la mullita.

En la tabla 1 se muestra la expresión analítica de la variación de $\Delta G^{\mathrm{o}}(T)$ de las siguientes reacciones: 


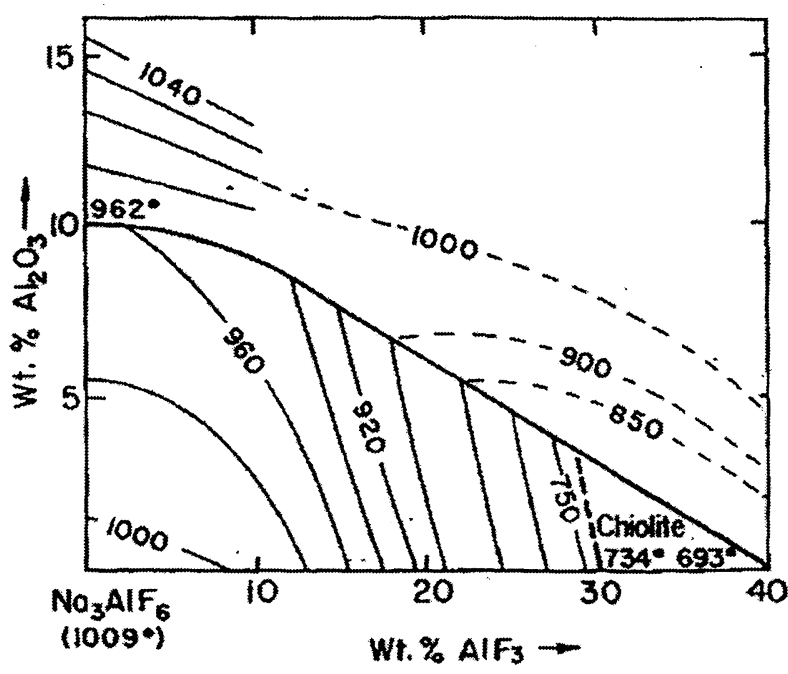

FIG. 2.- Diagrama de fases $\mathrm{Na}_{3} \mathrm{AlF}_{6}-\mathrm{AlF}_{3}-\mathrm{Al}_{2} \mathrm{O}_{3}$.

FIG. 2.- Phase diagram $\mathrm{Na}_{3} \mathrm{AlF}_{6}-\mathrm{AlF}_{3}-\mathrm{Al}_{2} \mathrm{O}_{3}$.

$$
\begin{aligned}
& \text { Re acción (5): } 3 \mathrm{SiC}_{(\mathrm{s})}+4 \mathrm{Al}_{(\mathrm{l})} \leftrightarrow 3 \mathrm{Si}_{(\mathrm{s})}+\mathrm{Al}_{4} \mathrm{C}_{3(\mathrm{~s})} \\
& \text { Re acción (6): } \mathrm{ZrO}_{2(\mathrm{~s})}+\mathrm{Al}_{(\mathrm{l})} \leftrightarrow 3 \mathrm{Zr}_{(\mathrm{s})}+2 \mathrm{Al}_{2} \mathrm{O}_{3(\mathrm{~s})}
\end{aligned}
$$

Aunque con respecto al aluminio la interacción con la cristobalita es la más favorable, las reacciones con la zircona y el carburo de silicio son posibles en menor extensión.

Todas las afirmaciones realizadas hasta el momento tienen un marcado carácter termodinámico. Sin embargo, desde el punto de vista cinético, no es lo mismo la velocidad de disolución de una alúmina mal cristalizada que la de un cristal de alúmina tabular.

\section{POSIBLES Alternativas. CONCLUSIONES}

Siendo inevitable la reacción entre el aluminio y los productos fluorados con los diferentes materiales refractarios, es posible esperar algún efecto beneficioso de los productos de corrosión sobre la velocidad de degradación del mismo (5 y 6).

Es posible aprovechar la formación de alúmina como producto de corrosión de la cristobalita para que una vez emulsionada dentro del aluminio pueda transitar hacia regiones más oxidantes y dar lugar a un compuesto de alúmina-aluminio de buena resistencia mecánica (7).

El análisis de los productos de corrosión del sistema $\mathrm{SiO}_{2}-\mathrm{Al}_{2} \mathrm{O}_{3}$ pueden resultar beneficiosos para el material:

- Formación de composites de alúmina-aluminio.
- Formación de costras criolita-alúmina-fluoruro de aluminio.

La extensión y la estabilidad de los productos de corrosión, además de su adherencia al refractario, son otras de las posibilidades a considerar en el diseño de una instalación. La formación de costras de corrosión, lejos de resultar perjudiciales, pueden llegar a ser beneficiosas y alargar la vida en servicio del revestimiento siempre y cuando se pueda controlar su estabilidad y crecimiento.

La temperatura en la intercara del refractariolíquido es la responsable de la desaparición-disolución de la costra o del crecimiento de la misma. Se necesita, por tanto, efectuar un diseño térmico del sistema con objeto de procurar una distribución uniforme de las temperaturas a lo largo de la intercara que impida la creación de zonas más frías.

La desaparición de la costra protectora puede provocar la aparición de un mecanismo de desgaste de alta eficacia: la abrasión de las partículas más refractarias. que, una vez desintegrada la costra, quedan emulsionadas en el líquido.

La formación de costras debidas a la interacción de productos fluorados y aluminio sobre refractarios del sistema sílice-alúmina puede verse favorecida si en la formulación del material, en lugar de responder con altos porcentajes de alúmina, se intenta aumentar el contenido de alcalinos.

El reciclado de materiales catódicos expuestos a la contaminación de productos fluorados puede ser una alternativa eficaz a la degradación de los materiales del sistema $\mathrm{SiO}_{2}-\mathrm{Al}_{2} \mathrm{O}_{3}$ por el aluminio y los productos fluorados (8).

La formulación de materiales no conformados aluminosos $\left(55 \% \mathrm{Al}_{2} \mathrm{O}_{3}\right)$ con altos porcentajes de sodio $\left(3 \% \mathrm{Na}_{2} \mathrm{O}\right)$ garantizan la formación de afinidades eutécticas $\left(1.050{ }^{\circ} \mathrm{C}\right.$ en el ternario mullita-tridimita-albita (Fig. 3)) a bajas temperaturas, provocando que la densidad del material puede acercarse a la real con la consiguiente disminución de la porosidad.

Si se impide la penetración del líquido cerrando la porosidad abierta y se facilita la adherencia de productos de corrosión, se podrá conseguir retardar el deterioro del material y aumentar el ciclo operativo de instalación.

\section{Agradecimiento}

Los autores expresan su agradecimiento por el soporte financiero a la CICYT (CICYT MAT971184) y a la FICYT (FICYT PA-MAT96-02). A esta última, también por la concesión de una beca predoctoral al Ldo. R. Corzo. 


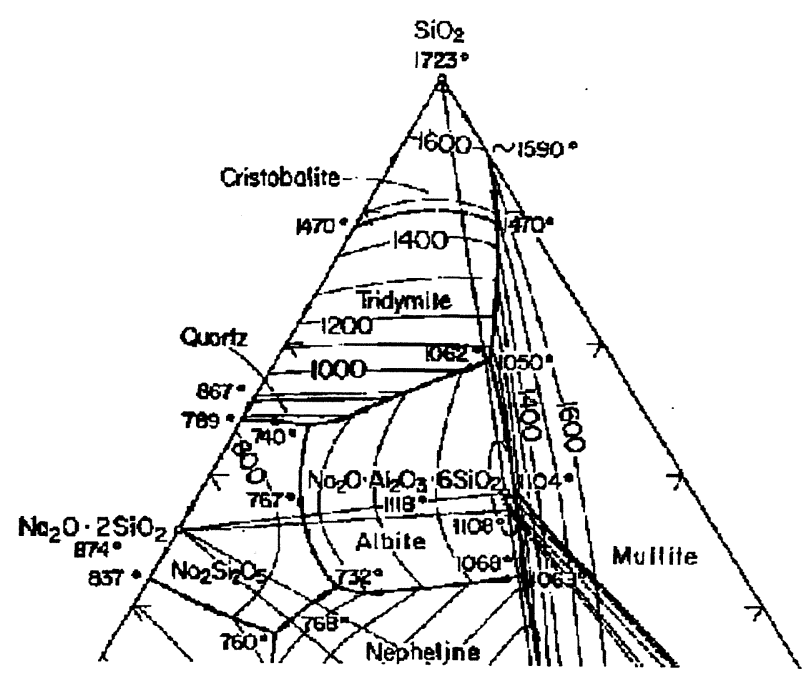

FIG. 3.- Diagrama ternario $\mathrm{SiO}_{2}-\mathrm{Al}_{2} \mathrm{O}_{3}-\mathrm{Na}_{2} \mathrm{O}$.

FIG. 3.- Ternary diagram $\mathrm{SiO}_{2}-\mathrm{Al}_{2} \mathrm{O}_{3}-\mathrm{Na}_{2} \mathrm{O}$.

\section{REFERENCIAS}

(1) Refractarios ALFRAN en la industria del aluminio y cobre. Fundidores. 53, 1997: 26-30.

(2) Bergeron, C. y Risbud, S. Introduction to phase equilibria in ceramics, $2^{a}$ ed. Am. Ceram. Soc. 1984.

(3) Kmacke, O., Kubaschewski, O. y Hesselmann, K. $2^{\mathrm{a}}$ ed. Springer-Verlag. Berlín (Alemania) 1991.

(4) Levin, M.F., Robbins, C.R. y McMurdie, H.F. Phase Diagrams for Ceramists. Am. Ceram. Soc., 1964 y 1969 suppl.

(5) BRABIE, V. Stahl Res. 68 (2), 1997: 54-60.

(6) Lachmund, H., Bannenberg, N., Bartiha, P. y Weibel, G. Stahl Eisen 117 (7), 1997: 69-74.

(7) Tai, W., Watari, T. y Torikai, T. Am. Ceram. Soc. Bull., 76 (4), 1997: 86-89.

(8) Blanco, F., Garcia, P., Verdeja, L.F., del Campo, J.J. y SANCHO, J.P. Rev. Minas, Oviedo 8, 1993: 77-88. 